\title{
Characterization of the Capillary Network in Skeletal Muscles From 3D Data
}

\author{
I. ERŽEN ${ }^{1}$, J. JANÁČEK ${ }^{2}$, L. KUBÍNOVÁ \\ ${ }^{1}$ Institute of Anatomy, Faculty of Medicine, University of Ljubljana, Ljubljana, Slovenia, \\ ${ }^{2}$ Department of Biomathematics, Institute of Physiology, Academy of Sciences of the Czech \\ Republic, v.v.i., Prague, Czech Republic
}

Received March 5, 2010

Accepted July 9, 2010

On-line October 15, 2010

\section{Summary}

In this review we present immunohistochemical methods for visualization of capillaries and muscle fibres in thick muscle sections. Special attention is paid to the procedures that preserve good morphology. Applying confocal microscopy and virtual 3D stereological grids, or tracing of capillaries in virtual reality, length of capillaries within a muscle volume or length of capillaries adjacent to a muscle fibre per fibre length, fibre surface area or fibre volume can be evaluated by an unbiased approach. Moreover, 3D models of capillaries and muscle fibres can be produced. Comparison of the developed methods with counting capillary profiles from 2D sections is discussed and the reader is warned that counting capillary profiles from $2 \mathrm{D}$ sections can underestimate the capillary length by as much as 75 percent. Application of the described 3D methodology is illustrated by the anatomical remodelling of capillarity during acute denervation and early reinnervation in the rat soleus and extensor digitorum longus muscles.

\section{Key words}

Capillaries • Skeletal muscle • Confocal microscopy • Stereology • 3D analysis $\cdot 3 \mathrm{D}$ visualization

\section{Corresponding author}

Ida Eržen, Institute of Anatomy, Faculty of Medicine, University of Ljubljana, Korytkova 2, SI-1000 Ljubljana, Slovenia. E-mail: ida.erzen@mf.uni-lj.si

\section{Introduction}

The capillary network composes the terminal part of the blood supply where the exchange of nutrients and metabolic waste products in individual cells occurs. Several physiological or experimental conditions either provoke growth of capillaries or cause degeneration and reduction in size (cf. Hudlická et al. 1992, Gute et al. 1994, Tyml et al. 1999, Borisov et al. 2000, Deveci et al. 2001, Hepple and Vogel 2004, Fujino et al. 2005).

In skeletal muscles the density of the capillary network has mostly been studied by counting capillary profiles from two-dimensional (2D) images of thin transverse sections (see Hudlická et al. 1992, Borisov et al. 2000, Harris 2005 for review).

However, capillaries are distributed in the threedimensional (3D) space, therefore the results obtained by counting capillary profiles from 2D transverse sections depend on 3D arrangement of capillaries and can substantially underestimate the capillary length, in special cases as much as 75 percent (Čebašek et al. 2010).

Our recent research was therefore dedicated to the methods that enable a clear-cut staining of capillaries and muscle fibres in thick muscle sections. Methodology is suited for stacks of optical images acquired by confocal microscopy. The obtained image data render 3D visualization of capillaries and muscle fibres in large muscle samples and enable unbiased and reproducible measurement of different parameters characterizing the capillary pattern in different muscles or in muscles 
undergone different pathological or experimental procedures. The 3D data may be also used in modelling of blood supply in skeletal muscle (Ji et al. 2006).

In this review we present an overview of our 3D approach to evaluation of capillarity of individual muscle fibres, including practical aspects of our methods. Application of the described 3D methodology is illustrated by the anatomical remodelling of capillarity during acute denervation and early reinnervation in the rat soleus and extensor digitorum longus muscles.

\section{Preparing the muscle samples for confocal microscopy}

Muscle samples were cut into $160 \mu \mathrm{m}$ thick cross sections with the cryotome Cryo-Star HM 560 (MICROM International GmbH; Walldorf, Germany). We had tested different fixatives and different staining protocols before we introduced a satisfactory staining of both capillaries and muscle fibre outlines. Moreover, special precautions were taken to protect muscle samples from mechanical injury during the staining and rinsing procedures. Finally, the stained sections had to be properly put to the slides without pressing the tissue.

It is very important that, immediately after cutting, tissue sections were put directly into medium which was kept at a constantly low temperature, e.g. in a mixture of water and ice. This prevented the tissue from extensive shrinkage (see also Dorph-Petersen et al. 2009).

A satisfactory staining was achieved by application of the triple immunofluorescent staining for CD31, laminin and fluorescein labelled Griffonia (Bandeira) simplicifolia lectin (Čebašek et al. 2004) (Figure 1A).

In accordance with published reports, some capillaries usually remained unstained, either with antibodies directed against the same antigen, or also with other staining protocols (cf. Paljärvi and Naukkarinen 1990). The triple combination is very efficient; nevertheless, we are aware that in some muscle samples the single lectin staining or double staining with lectin and laminin would be sufficient.

An important precondition for good penetration of antibodies is proper fixation and rinsing in buffered saline with addition of detergents like Triton X-100 or Tween. The recommended fixation time for $160 \mu \mathrm{m}$ thick sections should be at least 48 hours (for details see Čebašek et al. 2004, 2006, 2007). The dilution of primary antibodies should be adapted to different muscles and different experimental protocols. The dilutions applied in our studies are as follows: mouse anti rat CD31 (BD PharMingen) 1:3000, mouse anti human laminin (CHEMICON) 1:1000, fluorescein labelled Griffonia (Bandeira) simplicifolia lectin (GSL; Vector Laboratories) 1:300, goat anti mouse AlexaFluor 488 (Molecular Probes) 1:3000 and goat anti mouse AlexaFluor 546 (Molecular Probes) 1:3500. To achieve a more clear-cut staining we usually applied higher dilutions and longer incubation times (24 to 48 hours). Thick sections were incubated floating in the medium in special home-made baskets to protect the tissue from mechanical injury.

\section{Correction for shrinkage}

During the staining and mounting procedure muscle sample changes its dimensions, especially it shrinks along the $\mathrm{z}$-axis. The degree of shrinkage essentially affects the tortuosity and consequently the length of capillaries (Mathieu et al. 1983). To master this phenomenon we estimated the ratio between the nominal section thickness, set at the cryotome, and the real section thickness measured under the confocal microscope. The obtained value (between 3 and 4.35) was applied as the correction factor - the apparent distance between optical sections (axial calibration constant) had to be multiplied by this factor. The problem of muscle tissue deformation will be profoundly addressed in our next paper (in preparation). It will deal with vertical as well as with horizontal shrinkage of muscle samples that are finally observed under the confocal microscope.

\section{Parameters, characterizing capillarity in skeletal muscles}

In most studies on skeletal muscle capillary supply data are collected from 2D sections, by counting capillary profiles per fibre profile $(\mathrm{C} / \mathrm{F})$, per muscle cross-sectional area $(\mathrm{CD})$ or around a fibre profile (CAF). More sophisticated parameters like number of capillary contacts per fibre (CC), number of fibre contacts per capillary (FC), number of capillary contacts per fibre type area (CCFTA, i.e. the number of capillaries in contact with each muscle fibre, in relation to the type and area of the muscle fibre), the capillary-to-fibre ratio for each muscle fibre individually and the fibre perimeter (CFPE), local capillary to fibre ratio (LCFR) were applied in some studies only (for review see Hudlická et al. 1992, Degens 
et al. 1992, Lexell, 1997, Harris 2005, Čebašek et al. 2010). Actually, CAF represents the most frequent parameter used to characterize the capillary supply of skeletal muscles (Plyley and Groom, 1975, Plyley et al.
1998, Hepple and Mathieu-Costello 2001, MathieuCostello et al. 2005, Ryan et al. 2006, Wagatsuma 2008, Panisello et al. 2008).
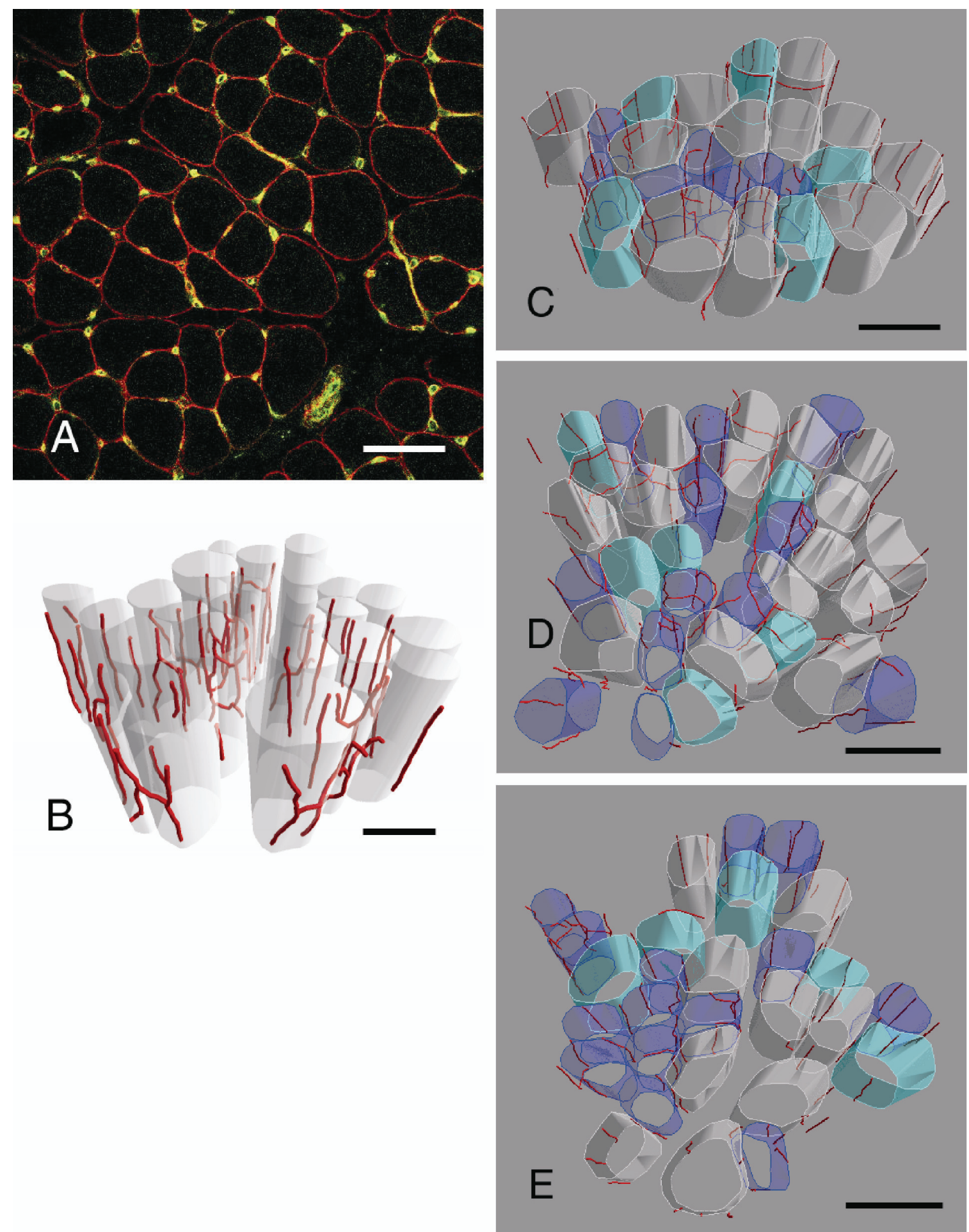

Fig. 1. Visualization of capillaries and muscle fibres in the extensor digitorum longus muscles of control, denervated and reinnervated rats. Staining of capillaries and muscle fibres in muscle samples, prepared for confocal microscopy (A). 3D visualization of capillaries and muscle fibres in control muscles (B). 3D visualization of capillaries and muscle fibre types in control (C), denervated (D) and reinnervated (E) rat extensor digitorum longus muscle. Fibre types were determined by the activity of the NADH-dehydrogenase: highly oxidative (dark blue), intermediate (turquoise), non-oxidative (white). Scale $=50 \mu \mathrm{m}$. 
Further, applying the stereological approach on 2D sections (Mathieu et al. 1983) the length of capillaries per unit muscle volume (Jv) was introduced, however, this approach needs to evaluate not only transversal but also longitudinal sections and it was only very rarely applied. Further, in human cricoarythenoid muscle, Lyon et al. (2007) estimated the length of capillaries per fibre and separately per each fibre type volume and surface area, using the orientator method (Mattfeldt et al. 1990). This method is unbiased, but demanding as it requires preparation of isotropic muscle sections, i.e. sections cut in various directions. Moreover, it is difficult to recognize capillaries adjacent to the given muscle fibre from oblique sections.

Our 3D approach (Kubínová and Janáček 1998, Kubínová et al. 2001, Janáček et al. 2009) collects information from stacks of microscopic images from transverse muscle sections and provides data on either length of capillaries per unit muscle volume (Lcap/Vmuscle), or length of capillaries, adjacent to individual muscle fibre per fibre length (Lcap/Lfib), fibre surface area (Lcap/Sfib) or fibre volume (Lcap/Vfib) (Čebašek et al. 2004, 2005, 2006, 2007, 2010).

\section{Quantitative methods for estimating capillarity in 3D}

Capillary network in skeletal muscles consists of capillaries that not only run parallel to the muscle fibre axis but decline from it, branch and form anastomoses, therefore their length estimation in $3 \mathrm{D}$ is more accurate than counting capillary profiles in 2D sections.

In our research we apply either 3D stereological computer generated grids that are inserted at random into stacks of optical sections (Kubínová et al. 2001) and count transsections with the capillaries, or we trace capillaries in the 3D space (Janáček et al. 2009).

\section{Stereological methods}

The length of capillaries supplying individual muscle fibres was estimated from systematically sampled stacks of optical sections by a ,global spatial sampling“ stereological method (Larsen et al. 1998) using slicer probes consisting of parallel equidistant test planes. Details of the method are explained elsewhere (Kubínová et al. 2001, Čebašek et al. 2006). Briefly, the length of capillaries (Lcap) adjacent (i.e. not farther than $5 \mu \mathrm{m}$ from the fibre surface) to individual fibres was estimated by counting intersections of the capillaries with a spatial grid consisting of three mutually perpendicular slicer probes with isotropic random direction using our Slicer plug-in module of the Ellipse program system (Tomori 2006) (Figure 2A-F).

The surface area of each muscle fibre in the given stack (Sfib) was estimated by counting intersections of the fibre surface with a cubic spatial grid consisting of three mutually perpendicular fakir probes, i.e., parallel test lines resembling nails of a fakir bed piercing the surface (Kubínová and Janáček 1998) using our Fakir plug-in module of the Ellipse program system (Figure 2G-I).

\section{Fig. 2. Analysis of the capillary network in skeletal muscles.}

Stereological measurement of Lcap using slicer probes $(\boldsymbol{A}-\boldsymbol{F})$. A. Spatial grid consisting of three mutually perpendicular slicer probes with isotropic random direction is applied to a capillary bed of individual muscle fibre and intersections (blue balls) of the capillaries and parallel test planes are counted (B-F). The intersections are marked during browsing through subsequent optical sections of the muscle. Here, three capillary intersections (blue dots) with the test planes (grey lines) are marked in five consecutive sections. A Slicer plug-in module of the Ellipse program system was used for this measurement. Scale $=20 \mu \mathrm{m}$. (Reprinted from Čebašek et al. 2010, with permission from Elsevier).

Stereological measurement of fibre surface area (Sfib) using slicer probes (G-I). G. A fakir probe consisting of parallel test lines is applied to the muscle fibre and intersections (blue balls) of the fibre surface and fakir probe are counted (H-I). The intersections are marked during browsing through subsequent optical sections of the muscle. Here, four intersections (blue dots) of fibre surface with the fakir probe are marked in two consecutive sections. A Fakir plug-in module of the Ellipse program system applying above measurement three times with three mutually perpendicular fakir probes was used. Scale $=20 \mu \mathrm{m}$.

Stereological measurement of fibre volume (Vfib) using the spatial grid of points (J-L). J. A spatial grid of points (blue balls) is applied to the muscle fibre and then points falling within the fibre are counted $(\mathrm{K}-\mathrm{L})$. The points falling into the fibre profiles are marked in serial optical sections of the muscle. Here, 14 points (in the centre of white crosses) are marked in both fibre sections that were $8 \mu \mathrm{m}$ apart. A PointGrid plug-in module of the Ellipse program was used for this measurement. Scale $=20 \mu \mathrm{m}$.

Tracing the capillaries in $2 D$ sections $(\boldsymbol{M})$. The sections of the image stack in three orthogonal directions are displayed. Capillary profile is marked interactively by computer mouse in any of the three views. Points marked in neighbouring planes are visible in either blue or yellow colour for the points below or above the image plane, respectively. Red lines denote the positions of the orthogonal planes. Scale $=50 \mu \mathrm{m}$.

$3 D$ visualization of capillaries in neighbourhood of a muscle fibre $(\boldsymbol{N})$. The surface of the neighbourhood is composed of triangles and rendered transparent. The capillaries are built of cylinders linked by balls with equal diameter. Also the contours of the muscle fibres at the bottom and topmost optical sections are shown. Scale $=50 \mu \mathrm{m}$. 

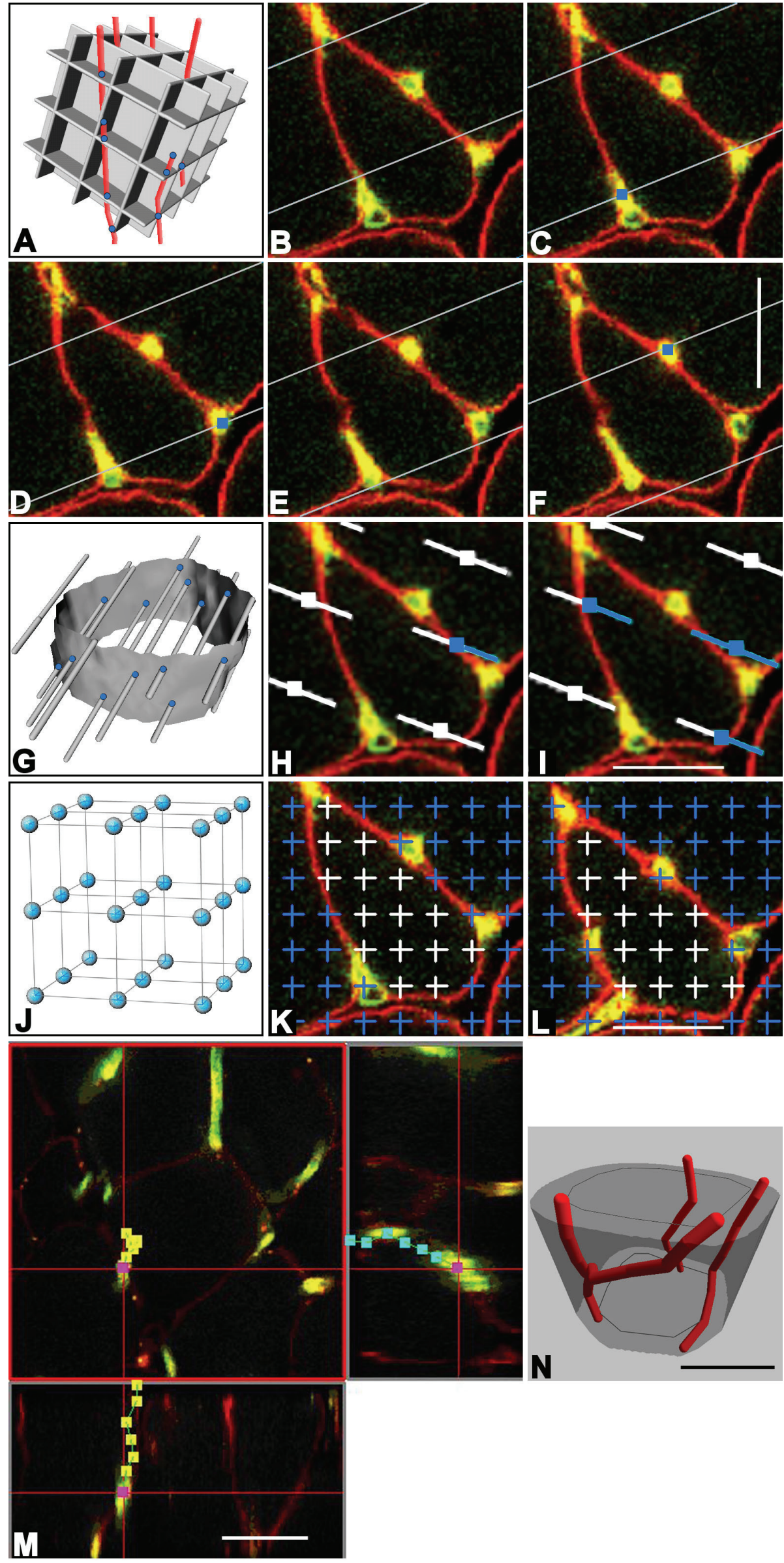
The volume of each muscle fibre in the given stack (Vfib) was estimated using the spatial grid of points (Cruz Orive 1997) where the test points falling within the fibre were counted using our PointGrid plug-in module of the Ellipse program system (Figure 2J-K).

\section{Image analysis}

The capillaries can be traced interactively in 3D using either 2D visualization of the confocal image stack in three orthogonal sections or using stereoscopic volume rendering and haptic device. As a result of the tracing procedure we obtain a representation of each capillary segment by a chain of line segments in 3D (Figure 2M). Useful geometrical characteristics of the segments can be calculated directly from this representation, e.g. the length of capillaries can be calculated by summing up the lengths of corresponding line segments.

The total length of the capillaries around each individual muscle fibre can be calculated by summing up the lengths of the line segments inside the fibre neighbourhood. The neighbourhood of the fibre can be represented by triangulated surface spanning contours obtained by dilation of contours of the fibre cross section in the topmost and bottom image (Figure 2N).

Many of the capillaries in contrast images can be detected automatically. First, we segment the capillaries by Gauss filter, top hat filter, thresholding of the image and skeletonizing the result by 6-pass algorithm by Palágyi (Palágyi and Kuba 1998). Then the chains of line segments of capillaries are obtained by tracing the thin lines voxels and connecting them to the centres of mass of adjacent thick parts. Finally, we resample the chains to obtain uniform length of segments. The uniform length must be small enough to represent the course of capillaries well but big enough to smooth the effect of digital image discretization - the automatic tracing yields representation of capillaries by short segments, longer than the actual length of capillaries.

\section{D-visualization of capillaries and muscle fibres}

The representation of the capillaries as chains of line segments and of the muscle fibres by triangulated surface spanning the outlines of the fibres, described above, can be used also for 3D visualization of the muscle. The capillaries can be visualized as chains of cylinders connected by balls and the fibre surfaces are rendered transparent (Figure $2 \mathrm{~N}$ ).
The Ellipse program was applied, which is a user-friendly commercial program for image processing and analysis on PC, equipped with MS Windows (Microsoft), produced by ViDiTo (Slovakia, Tomori 2006). It is equipped with modules for stereological analysis and 3D visualization developed by a co-author (J.J.) of this paper.

\section{Remodelling of the capillary network in rat soleus and extensor digitorum longus muscles during acute denervation and early reinnervation}

To illustrate the above described 3D methodology we summarize the results obtained from the experimental research on the anatomical remodelling of the capillary network in rat extensor digitorum longus and soleus muscles during acute denervation and early reinnervation (Čebašek et al. 2004, 2006, 2007, Janáček et al. 2009, Čebašek et al. 2010).

Data on acute denervation and early reinnervation on the capillary network in skeletal muscles are very limited, but the effect of long-lasting denervation on skeletal muscle fibres and their supplying capillaries have been studied extensively.

Long lasting denervation provokes degeneration of capillaries (Borisov et al. 2000, Scelsi et al. 2006) which diminishes the capillary length and lowers the capillary to fibre ratio by $88 \%$ during the first 7 months of denervation (Borisov et al. 2000). Capillary degeneration occurs quicker than loss of muscle fibres, whereby oxidative fibres are more resistant to this effect than the glycolytic ones (Borisov et al. 2000). Atrophy and fibre type transformation first affect the myosin heavy chain $2 b$ expressing fibres resulting in a shift towards slower myosin heavy chain isoforms and higher percentage of hybrid fibres (Jakubiec-Puka et al. 1990, Michel et al. 1996, Windish et al. 1998, Germinario et al. 2002, Patterson et al. 2006).

During acute denervation both slow and fast fibre types undergo equal atrophy in soleus, while in fast muscles fast fibres are affected to greater extent than the slow ones; hypertrophy during reinnervation is similar in both fibre types (Jaweed et al. 1975). Early reinnervation which starts between the first and the third week provokes hypertrophy of muscle fibres and clustering of fibre types. The postdenervational atrophy of muscle fibres is fully reversible if reinnervation starts during the first few months post denervation (Borisov et al. 2000). 
Large and Tyler (1985) reported that capillary area densities within the grouped regions of the reinnervated muscle were not significantly different from the supply to the same fibre type in intact contralateral muscle. $\mathrm{C} / \mathrm{F}$ for more glycolytic fibre types was lower than in intact muscle, while the values for more oxidative fibres agreed closely with the control values. A selective growth and loss of capillaries seemed to occur during reinnervation, adjusting capillary supply to meet changed metabolic demands of the individual fibres following regrouping.

\section{Experiments}

Fifteen adult Wistar rats (average body weight: $230 \pm 20 \mathrm{~g}$ ) were divided at random into three groups: denervated ( $\mathrm{D}$; nerve-cut), reinnervated (R; nerve-crush) and sham-operated (C; control). Surgery was performed under general anaesthesia with Rompun (2\% solution; Bayer, Germany) and Ketanest (10 mg/ml; Parke-Davis, Germany). In all three groups, the skin on the rat's back was cut over the left hip to expose the sciatic nerve. No further surgical procedures were performed in the control $\mathrm{C}$, and the wounds were closed with steel clips. In group $\mathrm{D}$ the left sciatic nerve was cut, while in group $\mathrm{R}$ the nerve was crushed for 1 minute with a serrated haemostat (Ribarič et al. 1991). In order to prevent reinnervation, a 2-cm segment was excised from the distal part of the cut sciatic nerve in group D. Control and experimental animals were kept isolated in very small cages where their movement was limited. Five animals per groups D and $\mathrm{C}$ were bled to death under deep ether anaesthesia on day 14 after the operation and five animals from group $\mathrm{R}$ on day 28 after the nerve lesion. The entire soleus (SOL) and extensor digitorum longus (EDL) muscle were excised from the left hind limb of the animals in all groups and weighed before being frozen in liquid nitrogen. Whole transverse sections of SOL and EDL muscle were cut in the middle of the muscle belly. The Veterinary Administration of the Ministry for Agriculture, Forestry and Food, Republic of Slovenia approved all experimental procedures (Permit 326-07285/99).

Capillary pattern adjacent to an average fibre type was studied in SOL and EDL in the three experimental groups, five animals per group. In control EDL muscle capillarity of the smallest and the largest fibres within the five muscles was estimated (i.e. 45 small fibres per muscle and 40 large muscle fibres per muscle, altogether 85 fibres per muscle).
Table 1. Capillary supply of the rat soleus (SOL) and extensor digitorum longus (EDL) muscles estimated by Lcap/Lfib, Lcap/Sfib Lcap/Vfib. In EDL data on the capillary network are presented also separately for the smallest and the largest fibres (see Čebašek et al. 2005 for details).

\begin{tabular}{lrrrr}
\hline & \multicolumn{2}{c}{ Muscles } & \multicolumn{2}{c}{ Fibres in EDL } \\
& SOL & EDL & Small & Large \\
\hline \multirow{2}{*}{-muscles } & $\mathbf{5}$ & $\mathbf{5}$ & $\mathbf{5}$ & $\mathbf{5}$ \\
Lcap $/$ Lfib & $\mathbf{6 . 9 1}$ & $\mathbf{4 . 6 0}$ & $\mathbf{4 . 3 1}$ & $\mathbf{4 . 8 4}$ \\
SEM & 0.70 & 0.47 & 0.29 & 0.73 \\
Lcap $/$ Sfib $\left[\mu \mathrm{\mu m}^{-1}\right]$ & $\mathbf{3 1 . 3 7}$ & $\mathbf{3 0 . 1 1}$ & $\mathbf{3 9 . 0 3}$ & $\mathbf{2 3 . 8 6}$ \\
SEM & 1.53 & 1.35 & 0.44 & 1.22 \\
Lcap $/$ Vfib $\left[\mu \mathrm{m}^{-2}\right]$ & $\mathbf{2 0 2 9 . 0 0}$ & $\mathbf{2 5 9 8 . 0 0}$ & $\mathbf{4 6 2 5 . 0 0}$ & $\mathbf{1 5 3 5 . 0 0}$ \\
SEM & 76.03 & 54.11 & 386.84 & 58.14 \\
& & & & \\
\hline
\end{tabular}

Fibre type pattern and capillarity in SOL compared to $E D L$

Rat SOL is a homogeneous oxidative slow twitch muscle composed mainly of type 1 fibres that express $\beta$ slow myosin heavy chain (MyHC) isoform. Type $2 \mathrm{a}$ fibres expressing MyHC-2a or hybrid fibres coexpressing MyHC-1 with MyHC-2a are present very rarely. EDL muscle is composed of at least four pure fibre types (type1, 2a, 2x and 2b), expressing either MyHC-1, MyHC-2a, MyHC-2x or MyHC-2b respectively, and hybrid fibres that co-express two or even three MyHC isoforms (Čebašek et al. 2005, 2007).

Although EDL muscle is classified as a fast glycolytic muscle, it contains oxidative and non-oxidative fibres that differ in their cross-sectional area: the smallest are predominantly oxidative and the largest predominantly glycolytic (Nemeth and Pette 1981, Egginton 1990, Hämäläinen and Pette 1993).

The density of the capillary network, estimated by Lcap/Lfib of an average fibre in soleus is larger and capillaries are more tortuous than in the EDL. In the EDL smaller fibres are more oxidative than the larger ones. We tested which of the introduced parameters shows eventual differences in capillary supply of the smallest and the largest fibres in the three experimental groups of the EDL muscle. The stereological analysis did not reveal any difference in Lcap/Lfib between these two fibre groups, while Lcap/Sfib and Lcap/Vfib were larger in small fibres than in large fibres (Table 1) (Čebašek et al. 2005).

On average 55 fibres per muscle in SOL and 45 small fibres and 40 large fibres per muscle in EDL were analyzed from each originally $160 \mu \mathrm{m}$ thick muscle section. 
Table 2. Capillary network in SOL and EDL muscles affected by acute denervation and early reinnervation.

\begin{tabular}{lrrrrrr}
\hline & \multicolumn{1}{c}{ SOL } & & & EDL & \\
& control & denervated & reinnervated & control & denervated & reinnervated \\
\hline N muscles & $\mathbf{5}$ & $\mathbf{5}$ & $\mathbf{5}$ & $\mathbf{5}$ & $\mathbf{5}$ & $\mathbf{5}$ \\
Lcap $/$ Lfib & $\mathbf{6 . 9 1}$ & $\mathbf{4 . 3 8}$ & $\mathbf{6 . 3 4}$ & $\mathbf{4 . 6 0}$ & $\mathbf{4 . 6 2}$ & $\mathbf{4 . 8 5}$ \\
SEM & 1.56 & 0.63 & 0.13 & 0.45 & 0.39 & 0.13 \\
Lcap $/$ Sfib $\left[\mu \mathrm{m}^{-1}\right]$ & $\mathbf{3 1 . 3 7}$ & $\mathbf{3 8 . 4 2}$ & $\mathbf{3 4 . 6 1}$ & $\mathbf{3 0 . 1 1}$ & $\mathbf{4 7 . 8 3}$ & $\mathbf{3 5 . 8 9}$ \\
SEM & 3.43 & 11.33 & 1.00 & 3.01 & 8.29 & 1.17 \\
Lcap $/$ Vfib $\left[\mu \mathrm{mm}^{-2}\right]$ & $\mathbf{2 0 2 9}$ & $\mathbf{3 2 7 0}$ & $\mathbf{2 5 3 6}$ & $\mathbf{2 5 9 8 . 0 0}$ & $\mathbf{4 6 0 0 . 0 0}$ & $\mathbf{3 6 1 6 . 0 0}$ \\
SEM & 76.03 & 24.15 & 11.18 & 54.00 & 235.00 & 262.00 \\
\hline
\end{tabular}

\section{Effect of denervation and early reinnervation}

Acute denervation affects capillary network in SOL and EDL muscle differently (Čebašek et al. 2006, 2007). In both muscles fibres atrophy during denervation, however, in SOL Lcap/Lfib is reduced whilst it remains unchanged in EDL (Table 2). In SOL denervation evidently affects the tortuosity of the capillary network, what can be deduced from 3D visualization. Enlarged Lcap/Sfib and Lcap/Vfib in denervated EDL is only due to the reduced fibre cross-sectional area. The reported different microvascular response of rat soleus and EDL muscles is consistent with the respose of rat soleus and gastrocnemius muscles in disuse (Tyml et al. 1999) and different training protocols (Gute et al. 1994).

In both reinnervated muscles the capillary pattern recovers and the estimated values for Lcap/Lfib and Lcap/Sfib become similar to those of the control muscles (Table 2).

In agreement with other studies (Michel et al. 1996, Windisch et al. 1998, Patterson et al. 2006) denervation and reinnervation affect mostly type $2 b$ and type $2 \mathrm{x}$ fibres, while type $2 \mathrm{a}$ and type 1 undergo only slight changes. The number of pure $2 b$ fibres is not affected during acute denervation, but it declines during early reinnervation where it renders a high percentage of hybrid fibres. Since Lcap/Lfib remains unchanged in EDL muscle in all experimental groups, we assume that no angiogenesis has occurred, however, it is not excluded that capillary network might have been reduced around some fibres and enlarged around others.

Nevertheless, our data indicate that Lcap/Lfib is genetically predetermined and is characteristic for a muscle type and not for a fibre type. Rat SOL and EDL remodel their capillary network in a different way. In the rat SOL Lcap/Lfib is larger than in EDL, due to higher tortuosity and the existence of anastomoses in the SOL. During acute denervation Lcap/Lfib in SOL in shortened; this is accompanied either by a lower number of anastomoses and/or by the straightening of the originally tortuous capillary course (Čebašek et al. 2006). Although tortuosity was not calculated in those muscle samples, the 3D visualizations show that the capillaries are less tortuous, and so this could be the reason for reduced Lcap/Lfib. During reinnervation the capillaries lengthen again: both, the changes in fibre size and capillary length contribute to the capillary length density adaptation. In contrast, in the EDL, the capillary network does not change significantly in the length of capillaries related to individual muscle fibres. In larger fibres, shorter diffusion distances are most probably established by adjustment of the fibre size (Čebašek et al. 2007). See Figure 1D-F for visualization of muscle fibre types and capillaries.

\section{Capillarity is remodelled by fibre size}

This caption deals with anatomical remodelling only. Physiological aspects were thoroughly considered elsewhere (Hudlická 1991, Egginton and Hudlická 2000, Badr et al. 2003, Egginton 2009). The decisive factor that provokes capillary growth is the increased blood flow and shear stress through capillaries supplying muscle fibres that undergo transformation (Hudlická 1991, Badr et al. 2003, Egginton 2009). Muscle fibre hypertrophy is always accompanied by capillary growth (Hudlická 1992) which usually starts around glycolytic fibres (Gray and Renkin 1978, Hudlická 1992, Badr et al. 2003) which are usually fibres with the largest diameter within a muscle.

Early studies supported the idea that muscle fibres with a high oxidative potential are generally associated with a denser capillary network (Romanul 


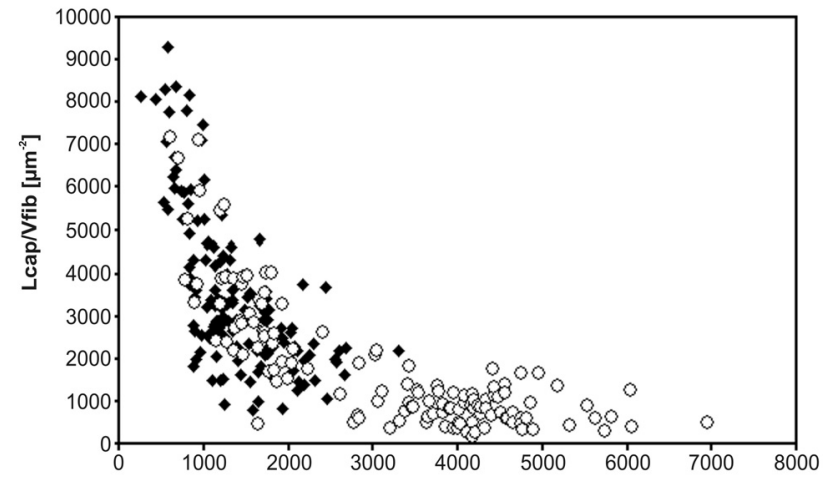

A

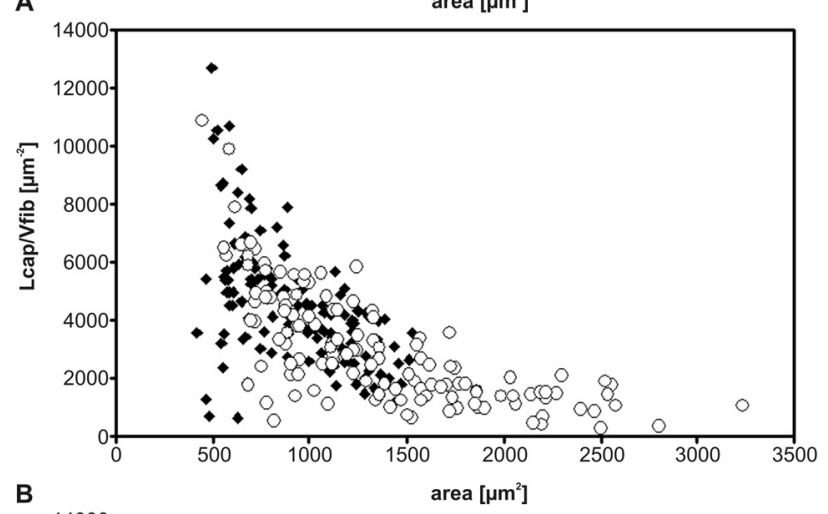

B

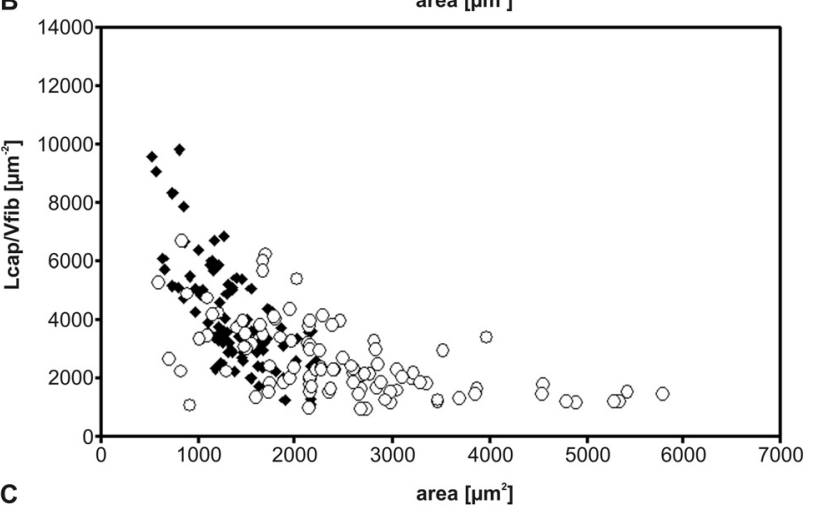

Fig. 3. Correlation between Lcap/Vfib and muscle fibre crosssectional area in extensor digitorum longus muscle from control (A), denervated (B) and reinnervated rats (C) (results for oxidative fibres are shown as solid squares, results for nonoxidative fibres as open circles).

1965, Romanul and Pollock 1969, Brown et al. 1976, Andersen and Henriksson 1977). This would mean that highly oxidative fibres require a higher rate of oxygen delivery than non-oxidative fibres; therefore, they must be better supplied with capillaries. However, conflicting results on this topic exist in the literature. According to the results obtained in rabbit (Gray and Renkin 1978, Ziada et al. 1984) and rat muscles (Large and Tyler 1985), capillary to fibre ratio for fast oxidative and slow oxidative fibres was larger than those for fast glycolytic fibres within the same muscle. Additionally, in muscles composed of fast glycolytic fibres, it was reported that capillary growth occurred without an increase in oxidative capacity or capillary growth preceded the increase in oxidative capacity (Cotter et al. 1973, Brown et al. 1976, Škorjanc et al. 1998, Egginton and Hudlická 2000) Moreover, Sillau (1985) estimated capillary to fibre ratio as well as mean and maximal diffusion distances in rat soleus and gastrocnemius muscles that underwent experimental hypothyroidism and reported lack of changes in capillarity despite the significant changes in oxidative capacity. Hepple and Vogel (2004) proved that the anatomic capillarity is maintained in relative excess of oxidative capacity in some muscle regions in late middle-aged rats. A further confirmation that the capillary supply of a fibre is primarily scaled according to fibre size and is relatively independent of the muscle fibre oxidative potential (Gray and Renkin, 1978, Large and Tyler, 1985, Ahmed et al. 1997, Wüst et al. 2009) was obtained from 3D data (Janáček et al. 2009) where Lcap/Lfib, Lcap/Sfib and Lcap/Vfib were estimated separately for capillaries adjacent to fibres with very high oxidative potential and those with very low oxidative potential in rat extensor digitorum longus muscle of control animals and those undergone acute denervation and early reinnervation respectively. In both fibre types, Lcap/Lfib was almost equal in all three animal groups. However, Lcap/Vfib was inversely related to the fibre cross-sectional area regardless the fibre type (Figure 3).

\section{Conclusion and further research}

Based on our research on the capillary estimation in $3 \mathrm{D}$ we strongly recommend the investigators in this field to critically consider all the conclusions based on results obtained by counting profiles in 2D (Čebašek et al. 2010) and keep in mind that those results could have been erroneous and misleading, especially in muscles with complicated capillary network. This implies human muscles, where capillaries are tortuous and form anastomoses. Further, skeletal muscle is a very plastic structure, able to adapt to intrinsic and extrinsic factors by fibre type transformation, changes in fibre size and in capillary pattern. Different parameters are available for the characterization of skeletal muscle capillarity. Those referring to muscle fibre size, i.e. muscle fibre crosssectional area, perimeter, surface area, or volume, might only reflect the adaptation in fibre size.

The most frequently published parameter CAF is 
easy to estimate and renders reasonable results on capillarity only when muscles with similar capillary arrangement are compared. Conversely, variable capillary arrangement can be satisfactorily assessed by the orientator method (Lyon et al. 2007) and 3D analysis, if a good staining is achieved and the results are corrected for shrinkage (this study). Additionally, the 3D analysis provides information on anastomoses, tortuosity and other parameters needed for modelling. In this respect our method is superior to the other ones as it provides additional relevant results.

Our further research aims at the combination of confocal microscopy with classical bright field microscopy, providing possibilities to assign capillaries to a histochemically and immunohistochemically defined fibre type, applying the newly developed software (Karen et al. 2009). This approach can open possibilities to study angiogenesis and vascular degeneration along histochemically defined fibre types by applying different markers for angiogenesis or degeneration and apoptosis.

\section{Conflict of Interest}

There is no conflict of interest.

\section{Acknowledgements}

This paper was financially supported by the Slovenian Research Agency and the Ministry of Education, Youth and Sports of the Czech Republic (KONTAKT grant No. MEB090910 and grant No. LC06063), and by the Academy of Sciences of the Czech Republic (grant AV0Z 50110509).

\section{References}

AHMED S, EGGINTON S, JAKEMAN PM, MANNION AF, ROSS H: Is human skeletal muscle capillary supply modelled according to fibre size or fibre type? Exp Physiol 82: 231-234, 1997.

ANDERSEN P, HENRIKSSON J: Capillary supply of quadriceps femoris muscle of man - adaptive response to exercise. J Physiol-London 270: 677-690, 1977.

BADR I, BROWN MD, EGGINTON S, HUDLICKÁ O, MILKIEWICZ M, VERHAEG J: Differences in local environment determine the site of physiological angiogenesis in rat skeletal muscle. Exp Physiol 88: 565-568, 2003.

BORISOV AB, HUANG S-K, CARLSON B: Remodelling of the vascular bed and prohressive loss of capillaries in denervated skeletal muscle. Anat Rec 258: 292-304, 2000.

BROWN M, COTTER MA, HUDLICKÁ O, VRBOVÁ G: The effects of different patterns of muscle activity on capillary density, mechanical properties and structure of slow and fast rabbit muscles. Pflugers Arch 361: 241$250,1976$.

ČEBAŠEK V, KUBÍNOVÁ L, RIBARIČ S, ERŽEN I: Capillary network in slow and fast muscles and in oxidative and glycolytic muscle fibres. Image Anal Stereol 24: 51-58, 2005.

ČEBAŠEK V, ERŽEN I, VYHNAL A, JANÁČEK J, RIBARIČ S, KUBÍNOVÁ L: The estimation error of skeletal muscle capillary supply is significantly reduced by 3D method. Microvasc Res 79: 40-46, 2010.

ČEBAŠEK V, KUBÍNOVÁ L, JANÁČEK J, RIBARIČ S, ERŽEN I: Adaptation of muscle fibre types and capillary network to acute denervation and shortlasting reinnervation. Cell Tissue Res 330: 279-289, 2007.

ČEBAŠEK V, KUBÍNOVÁ L, RIBARIČ S, ERŽEN I: A novel staining method for quantification and 3D visualisation of capillaries and muscle fibres. Eur J Histochem 48: 151-157, 2004.

ČEBAŠEK V, RADOCHOVA B, KUBÍNOVÁ L, RIBARIČ S, ERŽEN I: Nerve injury affects the capillary supply in rat slow and fast muscles differently. Cell Tissue Res 323: 305-312, 2006.

COTTER M, HUDLICKÁ O, PETTE D, STAUDTE H, VRBOVÁ G: Changes of capillary density and enzyme pattern in fast rabbit muscles during long-term stimulation. J Physiol-London 230: 34P-35P, 1973.

CRUZ-ORIVE LM: Stereology of single objects. J Microsc 186: 93-107, 1997.

DEGENS H, TUREK Z, HOOFD LJ, VAN'T HOF MA, BINKHORST RA: The relationship between capillarization and fibre types during compensatory hypertrophy of the plantaris muscle in the rat. J Anat 180: 455-463, 1992.

DEVECI D, MARSHAL JM, EGGINTON S: Relationship between capillary angiogenesis, fiber type, and fiber size in chronic systemic hypoxia. Am J Physiol Heart Circ Physiol 281: H241-H252, 2001. 
DORPH-PETERSEN K, CARIC D, SAGHAFI R, ZHANG W, SAMPSON AR, LEWIS DA: Volume and neuron number of the lateral geniculate nucleus in schizophrenia and mood disorders. Acta Neuropathol 117: 369-384, 2009.

EGGINTON S: Invited review: Activity- induced angiogenesis. Pflugers Arch 457: 963-977, 2009.

EGGINTON S: Numerical and areal density estimates of fiber type composition in a skeletal-muscle (rat extensor digitorum longus). J Anat 168: 73-80, 1990.

EGGINTON S, HUDLICKÁ O: Selective long-term electrical stimulation of fast glycolytic fibres increases capillary supply but not oxidative enzyme activity in rat skeletal muscles. Exp Physiol 85: 567-574, 2000.

FUJINO H, KOHZUKI H, TAKEDA I, KIYOOKA T, MIYASAKA T, MOHRI S, SHIMIZU J, KAJIYA F: Regression of capillary network in atrophied soleus muscle induced by hindlimb unweighting. J Appl Physiol 98: 1407-1413, 2005.

GERMINARIO E, ESPOSITO A, MEGIGHIAN A, MIDRIO M, BIRAL D, BETTO R, DANIELI-BETO D: Early changes of type 2B fibers after denervation of rat EDL skeletal muscle. J Appl Physiol 92: 2045-2052, 2002.

GRAY S, RENKIN EM: Microvascular supply in relation to fibre metabolic type in mixed skeletal muscles of rabbits. Microvasc Res 16: 406-425, 1978.

GUTE D, LAUGHLIN MH, AMANN JF: Regional changes in capillary supply in skeletal muscle of interval-sprint and low-intensity, endurance-trained rats. Microcirculation 1: 183-193, 1994.

HARRIS BA: The influence of endurance and resistance on muscle capillarization in elderly: a review. Acta Physiol Scand 185: 89-97, 2005.

HÄMÄLÄINEN N, PETTE D: Histochemical profiles of 3 fast fiber types in muscles of mouse, rat and rabbit. J Histochem Cytochem 41: 733-743, 1993.

HEPPLE RT, VOGEL JE: Anatomic capillarization is maintained in relative excess of fiber oxidative capacity in some skeletal muscles of late middle-aged rats. J Appl Physiol 96: 2257-2264, 2004.

HEPPLE R, MATHIEU-COSTELLO O: Estimating the size of the capillary-to-fiber interface in skeletal muscle: a comparison of methods. J Appl Physiol 91: 2150-2156, 2001.

HUDLICKÁ O: The response of muscle to enhanced and reduced activity. Bailliere's Clin Endocrinol Metab 4: $417-$ 439, 1990.

HUDLICKÁ O: What makes blood vessels grow? J Physiol London 444: 1-24, 1991.

HUDLICKÁ O, BROWN M, EGGINTON S: Angiogenesis in skeletal and cardiac muscle. Physiol Rev 72: 369-417, 1992.

JAKUBIEC-PUKA A, KORDOWSKA J, CATANI C, CARRARO U: Myosin heavy chain isoform composition in striated muscle after denervation and self-reinnervation. Eur J Biochem 193: 623-628, 1990.

JANÁČEK J, ČEBAŠEK V, KUBÍNOVÁ L, RIBARIČ S, ERŽEN I: 3D visualization and measurement of capillaries supplying metabolically different fiber types in the rat extensor digitorum longus muscle during denervation and reinnervation. J Histochem Cytochem 57: 437-447, 2009.

JAWEED MM, HERBISON GJ, DITUNNO J: Denervation and reinnervation of fast and slow muscles. A histochemical study in rats. J Histochem Cytochem 23: 808-827, 1975.

JI JW, TSOUKIAS NM, GOLDMAN D, POPEL AS: A computational model of oxygen transport in skeletal muscle for sprouting and splitting modes of angiogenesis. $J$ Theor Biol 241: 94-108, 2006.

KAREN P, ŠTEVANEC M, SMERDU V, CVETKO E, KUBÍNOVÁ L, ERŽEN I: Software for muscle fibre type classification and analysis. Eur J Histochem 53: 87-95, 2009.

KUBÍNOVÁ L, JANÁČEK J: Estimating surface area by the isotropic fakir method from thick slices cut in an arbitrary direction. J Microsc-Oxford 191: 201-211, 1998.

KUBÍNOVÁ L, JANÁČEK J, RIBARIČ S, ČEBAŠEK V, ERŽEN I: Three-dimensional study of the capillary supply of skeletal muscle fibres using confocal microscopy. J Muscle Res Cell Motil 22: 217-227, 2001.

LARGE J, TYLER K: Changes in capillary distribution in rat fast muscles following nerve crush and reinnervation. J Physiol London 362: 13-21, 1985.

LARSEN JO, GUNDERSEN HJ, NIELSEN J: Global spatial sampling with isotropic virtual planes: estimators of length density and total length in thick, arbitrarily oriented sections. J Microsc 191: 238-248, 1998. 
LEXELL J: Muscle capillarization: morphological and morphometrical analyses of biopsy samples. Muscle Nerve $\mathbf{2 0}$ (Suppl 5): S110-S112, 1997.

LYON MJ, STEER LM, MALMGREN LT: Stereological estimates indicate that aging does not alter the capillary length density in the human posterior cricoarytenoid muscle. J Appl Physiol 103: 1815-1823, 2007.

MATHIEU O, CRUZ-ORIVE LM, HOPPELER H, WEIBEL ER: Estimating length density and quantifying anisotropy in skeletal-muscle capillaries. J Microsc-Oxford 131: 131-146, 1983.

MATHIEU-COSTELLO O, JU Y, TREJO-MORALES M, CUI L: Greater capillary-fiber interface per fiber mitochondrial volume in skeletal muscles of old rats. $J$ Appl Physiol 99: 281-289, 2005.

MATTFELDT T, MALL G, GHAREHBAGHI H, MÖLLER P: Estimation of surface area and length with the orientator. J Microsc-Oxford 159: 301-317, 1990.

MICHEL R, CAMPBELL RJ, JASMIN BJ: Regulation of myosin heavy chain expression in adult rat hindlimb muscles during short-term paralysis: Comparison of denervation and tetrodotoxin-induced neural inactivation. FEBS Lett 391: 39-44, 1996.

NEMETH P, PETTE D: Succinate-dehydrogenase activity in fibers classified by myosin ATPase in 3 hindlimb muscles of rat. $J$ Physiol-London 320: 73-80, 1981.

PALÁGYI K, KUBA A: A 3D 6-subiteration thinning algorithm for extracting medial lines. Pattern Recognition Letters 19: 613-627, 1998.

PALJÄRVI L, NAUKKARINEN A: Histochemical method for simultaneous fiber typing and demonstration of capillaries in skeletal muscle. Histochemistry 93: 385-387, 1990.

PANISELLO P, TORRELLA JP, ESTEVA S, PAGES T, VISCOR G: Capillary supply, fibre types and fibre morphometry in rat tibialis anterior and diaphragm muscles after intermittent exposure to hypobaric hypoxia. Eur J Appl Physiol 103: 203-213, 2008.

PATTERSON M, STEPHENSON GMM, STEPHENSON DG: Denervation produces different single fiber phenotypes in fast- and slow-twitch hindlimb muscles of the rat. Am J Physiol-Cell Physiol 291: C518-C528, 2006.

PLYLEY M, GROOM A: Geometrical distribution of capillaries in mammalian striated-muscle. Am J Physiol 228: 1376-1383, 1975.

PLYLEY M, OLMSTEAD BJ, NOBLE EG: The time course of changes in capillarization in hypertrophied rat plantaris muscle. J Appl Physiol 84: 902-907, 1998.

RIBARIČ S, STEFANOVSKA A, BRZIN M, KOGOVŠEK M, KROŠELJ P: Biochemical, morphological, and functional-changes during peripheral-nerve regeneration. Mol Chem Neuropathol 15: 143-157, 1991.

ROMANUL F: Capillary supply and metabolism of muscle fibers. Arch Neurol 12: 497-509, 1965.

ROMANUL F, POLLOCK M: The parallelism of changes in oxidative metabolism and capillary supply of skeletal muscle fibers. In: Modern Neurology. S LOCKE (ed), Little Brown, Boston, 1969, vol 12, pp 203-213.

RYAN N, ZWETSLOOT KA, WESTERKAMP LM, HICKER RC, POFAHL WE, GAVIN TP: Lower skeletal muscle capillarization and VEGF expression in aged vs. young men. $J$ Appl Physiol 100: 178-185, 2006.

SCELSI R, POGGI P, CARRARO U, KERN H: Flaccid paraplegia: improvement of the muscle capillary supply after started daily functional electrical stimulation (FES) in human permanent lower motoneuron denervation. Basic Appl Myol 16: 105-107, 2006.

SILLAU A: Skeletal muscle capillarity in hyperthyroid and hypothyroid rats. Adv Exp Med Biol 191: 341-353, 1985.

S̆KORJANC D, JASCHINSKI F, HEINE G, PETTE D: Sequential increases in capillarization and mitochondrial enzymes in low-frequency-stimulated rabbit muscle. Am J Physiol - Cell Physiol 274: C810-C818, 1998.

TOMORI Z: Ellipse. Version 2.07. ViDiTo, Košice, 2006.

TYML K, MATHIEU-COSTELLO O, CHENG L, NOBLE EG: Differential microvascular response to disuse in rat hindlimb skeletal muscles. J Appl Physiol 87: 1496-1505, 1999.

WAGATSUMA A: Effect of hindlimb unweighting on expression of hypoxia-inducible factor-1 alpha, vascular endothelial growth factor, angiopoietin, and their receptors in mouse skeletal muscle. Physiol Res 57: 613-620, 2008.

WINDISCH A, GUNDERSEN K, SZABOLCS MJ, GRUBER H, LØMO T: Fast to slow transformation of denervated and electrically stimulated rat muscle. $J$ Physiol-London 510: 623-632, 1998. 
WÜST RCI, GIBBINGS SL, DEGENS H: Fiber capillary supply related to fiber size and oxidative capacity in human and rat skeletal muscle. Adv Exp Med Biol 645: 75-80, 2009.

ZIADA AMA, TYLER KR, WRIGHT AJA, HUDLICKÁ O: The effect of long-term vasodilatation on capillary growth and performance in rabbit heart and skeletal muscle. Cardiovasc Res 18: 724-732, 1984. 